\title{
Comparison of retention and extinction of a visual discrimination as an index of forgetting in mice
}

\author{
BÉATRICE ALESCIO-LAUTIER and BERNARD SOUMIREU-MOURAT \\ C.N.R.S., Université de Provence, Marseille, France
}

\begin{abstract}
The retention and extinction of a visual discrimination was examined in BALB/c mice. The mice were trained to perform a go/no-go discrimination task in parallel runways. Initial training resulted in an intermediate level of performance. Testing consisted of an additional session using either retention (Experiment 1) or extinction (Experiment 2) at one of five time intervals between 1 and 30 days. It was found in Experiment 1 that forgetting progressively increased over intervals of between 14 and 30 days. In Experiment 2, extinction testing induced more impairment of performance, so that forgetting occurred earlier relative to retention testing in Experiment 1 . However, in both experiments, the measure of performance by a discrimination ratio revealed the same amount of forgetting when the training-test interval was 30 days. These results define the forgetting curve for such a discrimination by mice. They are discussed in terms of possible factors involved in forgetting.
\end{abstract}

In studies concerned with animal memory processes, particularly in the study of long-term memory, the results obtained have not revealed substantial forgetting when short retention intervals were used (from a few hours to a few days long). Messenger (1971) and Holloway and Wansley (1973) reported in their experiments a multiphasic retention curve (i.e., with several inflections) with 24 to $72 \mathrm{~h}$ of delay after training. Thus, when short retention intervals were used, performance did not necessarily decrease directly over time.

When one or several long delays are used after training, the rate of forgetting seems to be related to numerous factors, among which the nature of the task is particularly important. Forgetting occurs under some conditions but not under others (for a review, see Gleitman, 1971). No forgetting was observed by Coulter, Collier, and Campbell (1976) with rats in a conditioned emotional response at 42 days of delay. Steinert, Infurna, and Spear (1980) reported similar results with a conditioned taste aversion: retention performance following a short ( 6 days) or a long ( 60 days) retention interval did not differ in adult rats. In both results, specific information related to stress and physiological disorders were present, and it is likely that, in these conditions, longer delays would be necessary for the appearance of forgetting. However, Sara (1984) reported reliable forgetting of a well-learned conditioned emotional response (passive avoidance) after a 25-day retention interval.

We wish to thank J. L. Martinez, Jr., for reading the English manuscript, and F. Roman for his help in developing the behavioral task in mice.

The authors' mailing address is: Laboratoire de Neurobiologie des Comportements, U.A. 372 CNRS, Université de Provence, Centre de St.-Jérome, 13397 Marseille Cedex 13, France.
Substantial forgetting of a visual discrimination task was found by Kraemer (1984) using a food-rewarded task with pigeons. Three different intervals were used, and the results showed that the amount of forgetting increased directly over intervals of 1,10 , and 20 days between training and retention test. According to Spear (1978), forgetting is typically regarded as an empirical construct, that is, as an observable decrement in performance of a learned response over time. Results reported by Kraemer (1984) agree with this finding.

Unfortunately, the majority of authors, in their experiments, have employed either several short retention intervals or only one long delay. As discussed above, when retention tests were carried out a few hours after training, many changes in performance were observed. Interpretation of these results is somewhat difficult and a few known assumptions predict these inflections only on the basis of mnemonic treatments. Therefore, in the study of forgetting, the use of only one delay is not sufficient, because performance observed at the retention test necessarily reflects the level of forgetting only for this delay. Thus, such results do not give any information about the waning of forgetting, that is, on the evolution of performance with time.

The present study was designed to provide a detailed examination of forgetting of successive visual discriminations by mice. After the initial training, retention (Experiment 1) and extinction (Experiment 2) were tested to better evaluate the level of performance and, consequently, the amount of forgetting.

\section{EXPERIMENT 1}

In Experiment 1, the forgetting of a go/no-go discrimination with food reinforcement was assessed in five differ- 
ent groups of mice at five retention intervals after training (from 1 day to 30 days). The discrimination task we used was similar to the one described by Brito, Thomas, and Stanton (1982). Initial training was defined for all groups so that each group displayed an intermediate level of learning. Thus, an increase or a decrease of performance could be observed in retention testing, depending on the delay.

Global performance was first considered for each learning session and for the retention test. However, this measure is not exact, because each of these sessions consisted of several trials. Indeed, there is considerable evidence to show that retention performance is widely determined by the degree of similarity between contextual learning cues and contextual retention cues. On the retention test, the first trial can be considered as involving combined contextual cues able to enhance memory retrieval. Therefore, in order to assess retrieval on the first trial and the effects of relearning within session, a trial-by-trial analysis was conducted.

\section{Method}

\section{Subjects}

The subjects were 50 male BALB/c mice from the Iffa-Credo Company; they were between 30 and 40 days of age when purchased. They were housed 20 per cage following arrival in the laboratory. They were maintained with food and water available ad lib under a 12-12 light-dark cycle (light on at $8 \mathrm{a} . \mathrm{m}$.) in a vivarium at temperatures of $21^{\circ}$ to $23^{\circ} \mathrm{C}$. All experimental procedures were conducted between 9 a.m. and 1 p.m. One week before the beginning of the experiment, the mice were housed individually and handled daily in order to decrease their emotional reaction.

\footnotetext{
Apparatus

Successive visual discrimination with food reinforcement was conducted in two side-by-side Plexiglas runways. Each alley was $5 \mathrm{~cm}$ wide and $67 \mathrm{~cm}$ long, and had side walls that were $15 \mathrm{~cm}$ high. At one end, a 9-cm-long startbox (SB) was separated from the alley by a guillotine door. At the other end, the goalbox (GB) consisted of a 5-mm-high cup on the floor at the end of the runway in an area that was delimited by a line on the floor $10 \mathrm{~cm}$ in front of the end of the alley. One alley (including the SB and GB) was black. The other alley (including the SB and GB) was white. The alleys were placed $10 \mathrm{~cm}$ center to center in the middle of a room with dim light.

\section{Procedure}

Habituation took place $48 \mathrm{~h}$ before learning. Each animal was successively placed for $3 \mathrm{~min}$ in each alley without any food, with a 30-sec interval between placements. Following habituation, the subjects were progressively food-deprived to between $80 \%$ and $85 \%$ of their free-feeding weights and given unlimited access to water. During training, they were weighed and fed daily $30 \mathrm{~min}$ after the learning session in order to maintain the same level of deprivation throughout learning.

Reinforcement consisted of one pellet $(10 \mathrm{mg})$ provided in the cup of the GB on S+ (go) trials. On S- (no-go) trials, no food was given. For half of the mice, reinforcement was delivered in the white runway; for the other half, food was provided in the black runway. The performances of the two groups with different $S+$ stimuli were similar during training. Consequently, they were combined for presentation of the results.
}

Each daily session consisted of 12 trials, half of which were S+ trials and half $S-$ trials. The sequence of $S+$ and $S-$ trials was randomized and differed in each training session. On each trial, a mouse was placed in the SB for $20 \mathrm{sec}$. Running time was measured with a stopwatch that was started when the door was opened following the $20 \mathrm{sec}$ and stopped either when the mouse crossed the line in front of the cup (four-paw criterion) or after $30 \mathrm{sec}$. Between each trial, the subject was put back in a waiting cage for $30 \mathrm{sec}$ in front of both runways. Initial training consisted of 1 session daily for 3 days. Depending on group (10 subjects in each group), testing consisted of an additional session, in the same conditions, at one of the five following intervals: $1,7,14,24$, and 30 days. In this retention test, the sequence of six $S+$ and six $S-$ trials was different from any of the initial sessions. For the 1-day interval, the subjects were fed as in initial training. For all the other intervals, the subjects were fed ad lib after the end of the third learning session; then they were food-deprived for $48 \mathrm{~h}$ before the test session.

\section{Results \\ Discriminative Learning \\ Performance was expressed as the mean of the running} times on the $\mathrm{S}+$ trials and the mean of the running times on the $S-$ trials for each group on a given session. Learning corresponded to a decrease of $S+$ running time and an increase of $S$ - running time, so that the difference between $\mathbf{S}+$ and $\mathbf{S}$ - running times, which represents the level of performance, increased as training progressed. The test session for the 1-day interval group (Figure 1) was the 4th day of learning. For this group, the difference between $\mathbf{S}+$ and $\mathbf{S}-$ trials was statistically significant by the second session $[t(9)>7.83, p<.001]$, and then increased in the following sessions. The scores in the third session could be considered as being at an intermediate level. We chose this level for the initial training and, in the different groups, the delay before the test session (retention control with food reinforcement) took place after the third session. Consequently, both improvement or reduction in performance could be detected in the following session as a result of the change in time of testing.

\section{Variable Test Intervals}

Initial learning. An analysis of variance on each day showed no statistical difference either for $S+$ running times $[F(4,45) \leq 2.47]$ or for $S$ - running times $[F(4,45)$ $\leq 2.39]$ for the five groups. Thus, all the five groups were pooled (Figure 1A). On the 1st day of learning, there was no statistical difference between $S+$ and $S$ - running times; however, $\mathbf{S}+$ and $\mathbf{S}$ - running times were different on the 2 nd day $[t(49)=10.47, p<.001]$.

Retention performance. Results are summarized in Figure 1B. An analysis of variance was performed on the mean $\mathbf{S}+$ and $\mathbf{S}-$ running times according to the retention interval. This analysis revealed a significant effect for the retention interval $[F(4,45)=9.96, p<.001]$, and the reinforcement $\times$ retention interval interaction was significant $[F(4,45)=12.61, p<.001]$. When the analysis was carried out on separate $S+$ and $S-$ running times, significant effects were observed for both $\mathrm{S}+$ running times $[F(4,45)=36.72, p<.001]$ and $S-$ running times 

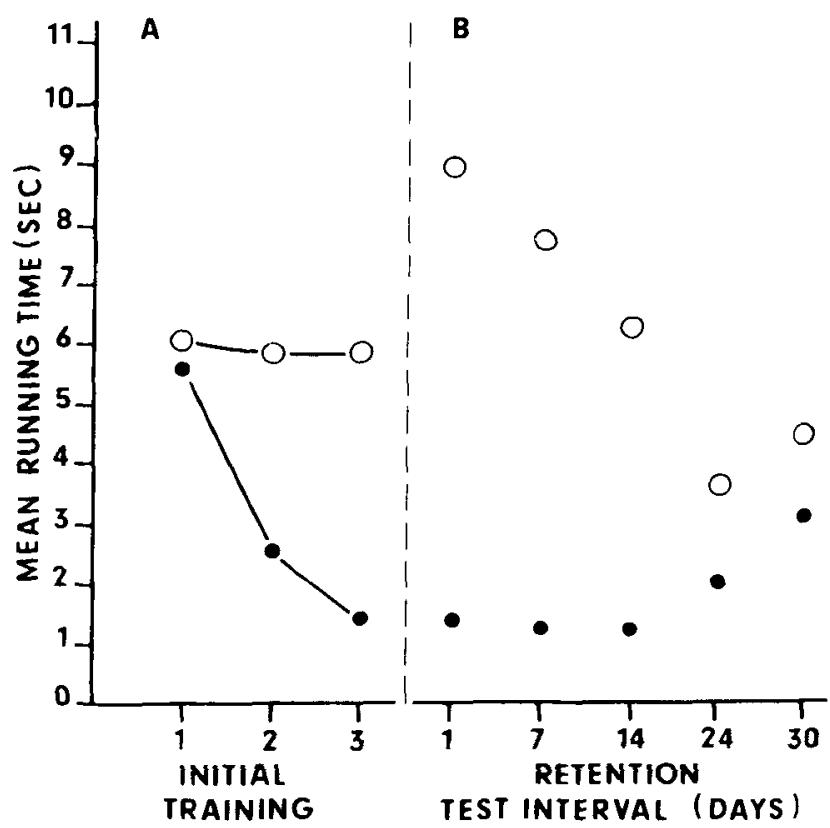

Figure 1. Mean $S+(\bullet)$ and $S-(O)$ running times per session. Panel A: Performances of all the subjects for the three sessions of initial training. Panel B. Performances on retention testing for each of five intervals of 1 to 30 days.

$[F(4,45)=4.23, p<.01]$. As shown in Figure 1B, the difference between $S+$ running times and $S-$ running times was progressively reduced from the 1-day interval to the 30-day interval, even though the difference was still statistically significant at this long interval $[t(9)=2.7$, $p<.05]$. Performance on the delayed test sessions $(7$ days to 30 days) was compared with that of the 1-dayinterval group. This allowed for an exclusive test of the influence of the time interval, apart from the effect on performance of a new learning session. Using the 7-day or 14-day intervals, no change was observed in the S+ running time, and the $S$ - running time was moderately reduced relative to the 1-day interval. However, these differences were not statistically significant. A clear deficit appeared only after the 14-day interval. More dramatic changes appeared at the 24-day and 30-day intervals. For both time intervals, as compared with the homologous values on the 1-day interval, $S+$ running times were progressively increased and $S-$ running times were strongly reduced $[t(18) \geq 3.07, p<.01]$.

To better illustrate the evolution of performance in relation to the time interval before testing, we defined a more global measure than separate $S+$ and $S$ - running times. We chose a discrimination ratio defined for a given session and for each subject as $\Delta=\Sigma(\mathbf{S}+) /[\Sigma(\mathbf{S}+)+$ $\Sigma(\mathrm{S}-)]$. On each day, the mean discrimination ratio for each group was based on ratios calculated for each mouse separately. Figure 2 presents this ratio for each of the five test sessions. It must be noted that a ratio of .50 corresponded to similar $\mathbf{S}+$ and $\mathbf{S}-$ running times. In other words, the smaller the ratio, the better the performance.
As can be seen in Figure 2, retention performance decreased after the 7-day interval. Statistical analysis showed that the retention interval was a significant factor $[F(4,45)$ $=63.21, p<.001]$. The largest amount of decay took place between the 14th and 24th days $[t(18)=7.51, p<$ $.001]$. This retention curve can be considered as illustrating the normal decay of performance over time, that is, forgetting (see Discussion).

In the present successive discrimination, each daily session included 12 trials. The above analysis of data provided a global measure of daily performance. However, due to the intermediate level of performance after initial training, a learning effect might have developed within a test session. In other words, a subject could improve its response relative to the previous trial, but the mean $\mathrm{S}+$ or $\mathrm{S}-$ running times might not have correctly reflected the level of performance. Therefore, we analyzed each test session trial by trial, considering the six successive $\mathrm{S}+$ and six successive $\mathrm{S}-$ running times (Figure 3). As had been observed for the global scores, a difference could be observed in the evolution within session when the three shortest time intervals were compared with the two longest ones. An analysis of variance was performed on the mean $S+$ and $S-$ running times. The factors included retention interval and trials within the test session. Significant effects were obtained for retention in-

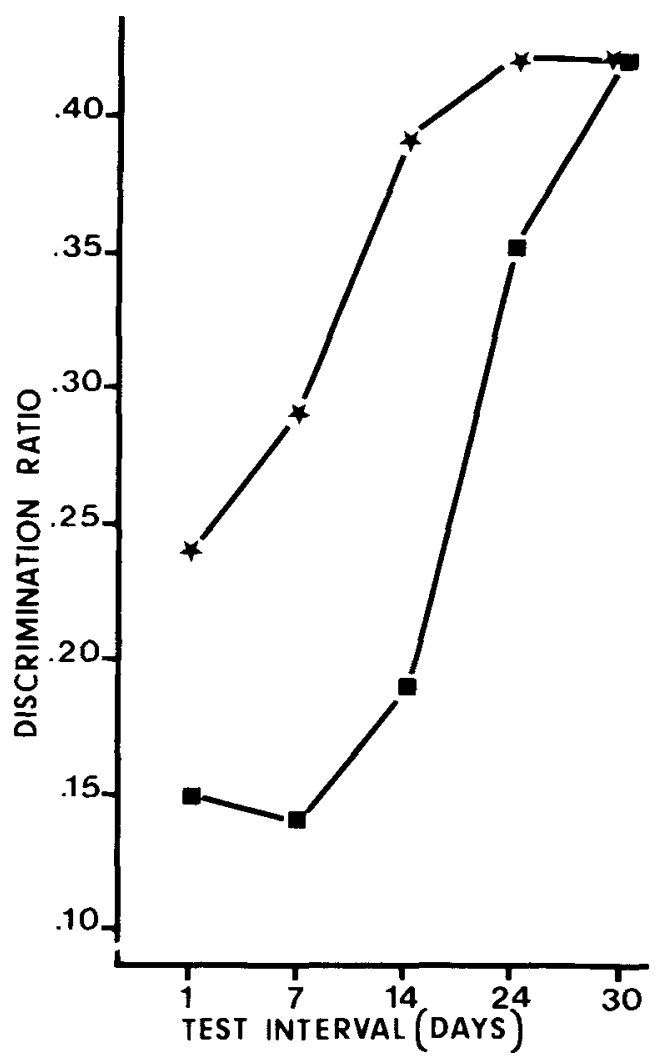

Figure 2. Discrimination ratio on the test session as a function of the time interval between initial training and retention $(\square-b)$ or extinction $(\star-\star)$ testing. 


\section{RETENTION TEST INTERVAL}

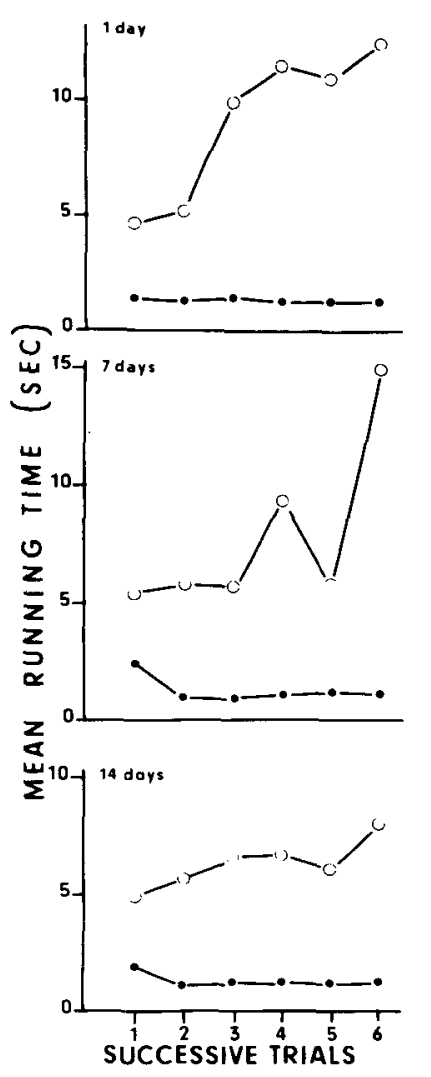

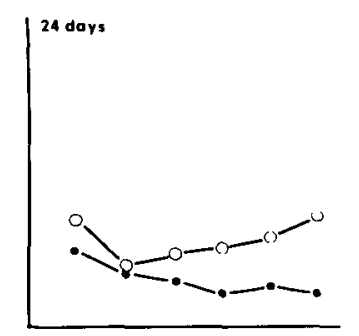
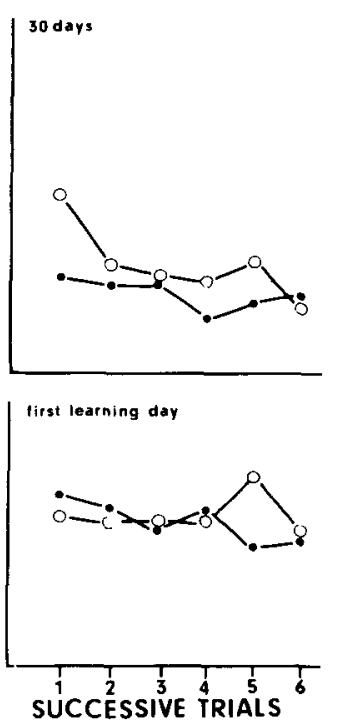

Figure 3. Trial-by-trial analysis of mean $S+(\theta)$ and $S-(O)$ running times during retention testing on each of five intervals between training and testing: $1,7,14,24$, or 30 days. The bottom panel on the right shows a similar analysis for the 1st day of initial training of the 50 subjects.

terval $[F(4,45)=9.96, p<.001]$ and for trials $[F(5,225)$ $=3.95, p<.005]$. Relearning appears to decrease over intervals. The trials $\times$ retention interval interaction was significant $[F(20,225)=3.93, p<.001]$. Considering the 1-day, 7-day, and 14-day intervals, the first $S+$ running time and the first $S$ - running time were statistically different for each of the three intervals $[t(9) \geq 5.46$, $p<.001$, even though they did not reflect the performance difference throughout the whole session. For these time intervals, subjects improved $\mathrm{S}-$ running time within the test session from the first to the last trial. Almost no change was observed in $S+$ running times.

The 24-day interval produced a different set of changes. No difference was observed between $\mathrm{S}+$ and $\mathrm{S}-$ running times on the two first trials $[t(9) \leq 2.10]$. S- running time increased only slightly over trials, but $S+$ running time revealed a progressive decrease so that the difference between $\mathbf{S}+$ and $\mathbf{S}$ - running times was statistically significant on the last four trials $[t(9) \geq 2.32, p<.05]$. On the test session after the 30-day interval, no significant difference was observed between $\mathrm{S}+$ and $\mathrm{S}-$ running times regardless of the trial $[t(9) \leq 1.83]$. It seems

that no learning within the session was possible, due to the poor level of retention at the beginning of the session. Moreover, the 30-day-interval group's performance was similar to that of naive subjects' on the first learning day (Figure 3).

\section{Discussion}

Taken together, these results showed a progressive decay of retention of a visual discrimination over time in mice. Forgetting progressively increased over intervals of $1,7,10,24$, and 30 days. Furthermore, relearning was possible only between 1 and 14 days after learning. Relearning was still present, though slight, after 24 days; it was undetectable after 30 days. Other findings are in agreement with our results. Kraemer (1984) reported that pigeons increasingly forget over time; Gleitman (1971) observed forgetting in rats, as measured by run time in a straight alley; and Deweer, Sara, and Hars (1980) demonstrated forgetting of a complex maze in rats after a training-test interval of 25 days. However, the appearance of forgetting is not so commonly evident (Spear, 1978). In fact, the amount of forgetting is controlled by several factors: nature of the task, level of training, time of testing and so forth. In our conditions, initial training produced an intermediate level of performance. Considering the retention test situation, the 1-day interval and even the 7-day interval can, in fact, be considered as additional learning sessions. Regarding intervals of 1 to 14 days, the observed changes in performance were attributable exclusively to changes in S- running times. Forgetting truly appeared only when the mice were tested 24 days later, inasmuch as the level of performance was then lower than it had been on the 3rd day of training. A marked decrement appeared after a 30-day interval. However, it is not clear whether or not forgetting was total for this interval.

The structure of the present task did not allow for a onetrial test situation. Several points are noted about repeated trials within the test session. An influence of the sequence of S+ and S- trials does not seem to be involved in our results, although others have observed such an influence (e.g., Haggbloom, 1980). Indeed, the sequence on the test session was the same whatever the time interval, and performance after 7 to 30 days was compared with the performance of the 1-day group (which had the same sequence). The improvement in performance found when testing the 1-day and the 7-day groups did not reflect the reminiscence that had been observed in mice after partial learning of appetitive operant conditioning (Jaffard, Destrade, Soumireu-Mourat, \& Cardo, 1974); the improvement in the present case was only the result of additional learning, as noted above. Indeed, the comparison of the first $\mathrm{S}+$ and $\mathrm{S}$ - running times for these intervals with performance on the third training session shows that there was no improvement at the beginning of the test session. The subjects apparently adapted their behavior over trials and improved their performance within the test session. For the longest intervals, the results are quite different. 
On the first trials, $\mathrm{S}+$ running times increased and then decreased within the session. Furthermore, $S-$ running times were also responsible for the performance decrement on the test session. Moreover, $\mathrm{S}-$ trials revealed a weak (24-day interval) or no (30-day interval) improvement over trials. Finally, no relearning could be seen in the 30-day group, even in the last trials of the test session. Thus, it appears that the forgetting is quite evident 30 days after an incomplete learning of a visual discrimination. Even so, the global performance on the test session revealed some residual level of learning.

A lot of data have proved the importance of context in the retrieval of memory (Spear, 1978). Reminders improve performance of animals that have "forgotten" either spontaneously (Deweer et al., 1980) or after an amnesic treatment (Sara, 1973). Also, any change in contextual cues can affect retention (Thomas, McKelvie, Ranney, \& Moye, 1981). No contextual influence seems to explain our data. Indeed, the experimental conditions were strictly the same during the initial training and the test session. It is unlikely that external cues might be responsible for any of the observed effects. Nevertheless, the first $\mathbf{S}+$ and $\mathbf{S}$ - trials could have acted as reminders that provided intratask cues on the following trials. In any case, the first $\mathrm{S}+$ and $\mathrm{S}-$ trials did not constitute reminders for the longest interval, because no improvement of performance was observed at the end of the session. On the other hand, food deprivation induced a similar level of motivation before both training and test sessions. This could act as an internal stimulus for retrieval, but it was not sufficient by itself, since performance revealed a marked decrement after 14 days. Finally, all of these cues may explain some of the performance on the first trials of the test session for the 7-day and 14-day groups, if considered together. Alternatively, for the longest intervals, a decrease in the efficiency of external and internal contextual cues could at least partially be involved in the decrement in performance. The observed deficits might be related to an increasing difficulty to extract or combine pertinent attributes of the task.

In addition, concerning the intermediate test intervals, there was a slight deficit of the $\mathrm{S}+$ stimulus at the beginning of the test session. The reduction in $S-$ running time in the early trials reflects a partial loss of inhibitory control by the $\mathbf{S}-$ stimulus. This could mean a weak forgetting of the stimulus situation at the beginning of the session. Then both $S+$ and $S-$ performance improved within the test session. With long retention intervals, a total loss of inhibitory control by the $S$ - was observed. This change in performance seems to be due to generalization processes. Nevertheless, retention of the response is still correct. In other words, the stimulus features were not forgotten at the same rate as the response itself. Thomas and Lopez (1962) observed that, when long retention intervals were used, stimulus generalization gradients were flattened, whereas retention of the response was preserved. However, it is difficult to discuss our study in terms of stimulus generalization gradients, because we did not manipulate the stimulus features.

In conclusion, our results seem to indicate a weakening of information processing for the longest intervals. This would correspond to the disinhibitory effect observed by Brito et al. (1982). In our conditions, inhibitory processes related to $S+$ and $S$ - values were preferentially altered over time and would be responsible for forgetting.

\section{EXPERIMENT 2}

In Experiment 2, the test session was run under extinction. Such a paradigm could influence the rate of forgetting observed in Experiment 1 in different ways. At first, the change between training and test session could induce interferences responsible for faster forgetting. Moreover, it could be expected that the lack of reinforcement would not allow for any relearning. And, finally, extinction considered as new learning was expected to influence performance in the same way as the forgetting of the previous situation.

The comparison of results for each of the five test intervals between both experiments might allow for an analysis of the roles of these factors. As in Experiment 1, global performance will be considered at first; then, to assess the above assumptions, a trial-by-trial analysis will be done.

\section{Subjects \\ Method \\ The subjects were 50 male $B A L B / c$ mice, housed in the same conditions as in Experiment 1.}

\section{Apparatus and Procedure}

The apparatus and the whole procedure (groups with different test intervals) were exactly the same as in Experiment 1, except that food reinforcement was not available during the test session (fourth session). In other words, the test session was an extinction session, with all the other conditions as previously described.

\section{Initial Training}

\section{Results}

An analysis of variance on each day showed no statistical difference either for $S+$ running times $[F(4,45) \leq$ $1.47]$ or for $S-$ running times $[F(4,45) \leq 2.38]$ for the five groups. As in Experiment 1, all the five groups were pooled (Figure 4A). There was no statistical difference between S+ and S- running times on the 1st day of learning, but a statistically significant difference appeared on the 2 nd and the 3 rd days $[t(49) \geq 12.46, p<.001]$.

\section{Extinction Performance}

Globally, performance was progressively impaired over sessions, mainly from the 14-day interval (Figure 4B). An analysis of variance was performed on the mean $\mathrm{S}+$ and $S$ - running times according to the retention interval. This analysis revealed a significant effect for the retention interval factor $[F(4,45)=3.35, p<.025]$. When 

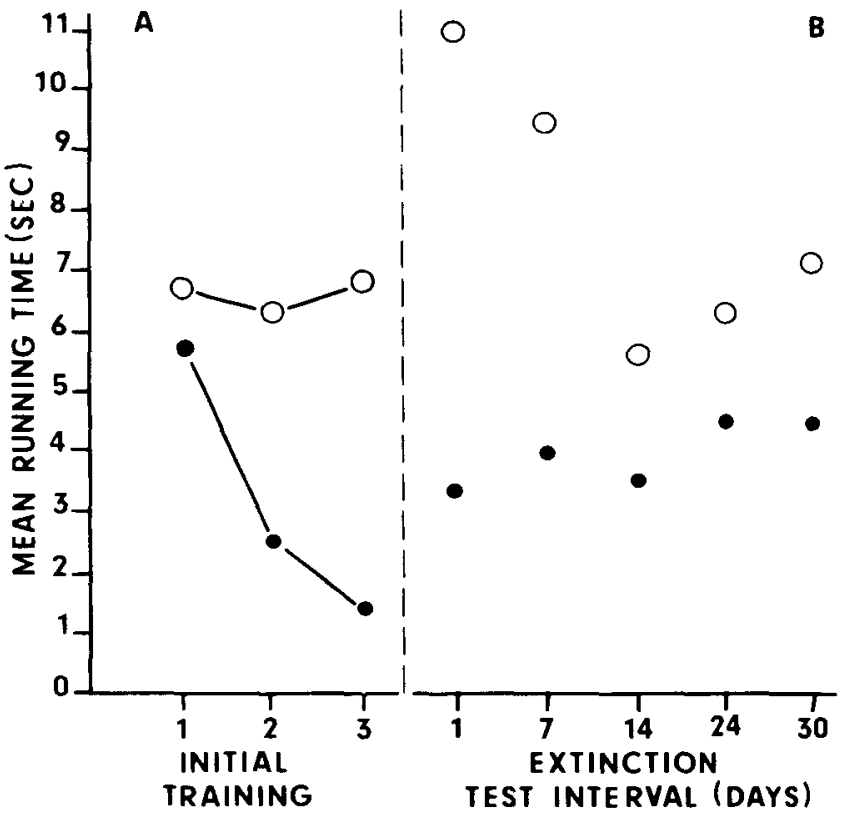

Figure 4. Mean $S+(O)$ and $S-(O)$ running times per session. Panel A: Performances of all the subjects for the three sessions of initial training. Panel B: Performances on extinction testing for each of five intervals of 1 to 30 days.

the analysis was carried out separately on $\mathrm{S}+$ and $\mathrm{S}-$ running times, no significant effect was observed for $\mathrm{S}+$ running times $[F(4,45)=0.53]$. As shown in Figure $4 \mathrm{~B}, \mathrm{~S}+$ running times did not change over retention interval, but a significant effect was observed for $\mathrm{S}$ - running times $[F(4,45)=4.92, p<.01]$. On the two first intervals, the main feature was an impairment of $S+$ running times. From the 14-day to the 30-day intervals, both S+ and Srunning times were affected by the impairment. The development of performance was relatively different from that found in Experiment 1. An accentuation of the deficit related to the test interval could be observed, especially at the 14-day interval. An impairment on S+ running times appeared as early as the 1-day interval (instead of the 24day interval in retention). S- running times on extinction were similar to corresponding values on retention for the two first intervals and were different at the 14-day interval. In any case, the difference between S+ and Srunning times was still statistically significant for the 30day extinction test $[t(9)=2.27, p<.05]$.

In considering the discrimination ratio as in Experiment 1 (Figure 2), it was found that extinction performance was worse for each of the test intervals than it was in the retention situation, starting from the 1-day test interval. However, this extinction performance showed a progressive impairment between sessions. At the 30-day test interval, both situations produced the same discrimination ratios, thus revealing residual learning as described for the comparison made between $\mathrm{S}+$ and $\mathrm{S}-$ running times in both situations.
We also examined performance within session, trial by trial, for each test interval (Figure 5). An analysis of variance was performed on the mean $S+$ and $S-$ running times. The factors included retention interval and trials within the test session. Significant effects were obtained for the retention interval $[F(4,45)=3.35, p<.025]$ and for trials $[F(5,225)=11.61, p<.001]$. For the 1-day interval, a tendency toward a slight progressive increase could be observed for $\mathrm{S}+$ running times. For this inter$\mathrm{val}$, the first $\mathbf{S}+$ running times and $\mathbf{S}$ - running times were statistically different $[t(9)=4.60, p<.01]$. This difference progressively increased through trials, even though it was paradoxical in view of the fact that the test situation was extinction (see Discussion). The same tendencies were observed using the 7-day interval. Contrary to Experiment 1, the deficit was clearly evident with extinction being used after a 14-day interval: the $S-$ running times strongly decreased and, at the same time, the S+ running times strongly increased on the last trials, so that the differences between both running times throughout the

\section{EXTINCTION TEST INTERVAL}

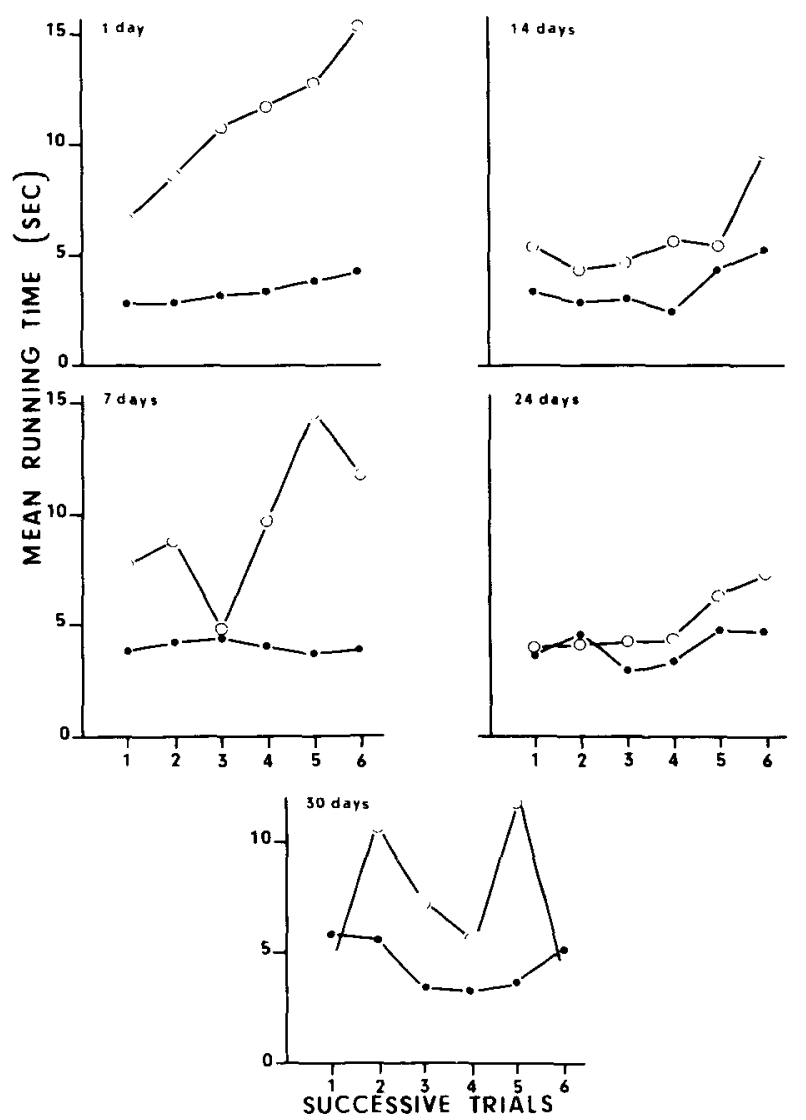

Figure 5. Trial-by-trial analysis of mean $S+(\theta)$ and $S-(O)$ running times during extinction testing on each of five intervals between training and testing: $1,7,14,24$, or 30 days. 
Table 1

Mean S+ and $\mathbf{S}-$ Running Times (in Seconds) Within Extinction Test Session After a 14-Day Interval as a Function of the Ordinal Position of the Trial

\begin{tabular}{lrrrrrr}
\hline & \multicolumn{7}{c}{ Trials } \\
\cline { 2 - 7 } & 1 & 2 & 3 & 4 & \multicolumn{1}{c}{5} & \multicolumn{1}{c}{6} \\
\hline $\mathrm{S}+$ & 3.41 & 2.90 & 3.04 & 2.48 & 4.32 & 5.17 \\
$\mathrm{~S}-$ & 5.45 & 4.16 & 4.65 & 5.53 & 5.46 & 9.26 \\
$t(9)$ & 2.58 & 2.58 & 1.48 & 2.33 & .87 & 2.97 \\
& $<.05$ & $<.05$ & n.s. & $<.05$ & n.s. & $<.02$ \\
\hline
\end{tabular}

Note-Statistics were carried out on the difference between $\mathrm{S}+$ and $\mathrm{S}-$ trials.

session were not all statistically significant (Table 1). A more important deficit was revealed after 24 days within the session, since $S+$ and $S-$ running times were not significantly different $[t(9) \leq 1.83]$. The same lack of significance was observed for the 30-day test interval [ $t(9)$ $\leq 1.75]$.

\section{Discussion}

A progressive decrease in performance was also observed with extinction. This decrease was more marked for each interval in Experiment 2 than had been observed in Experiment 1. Apart from the influence of the time interval, the absence of reinforcement represented a change in the paradigm, so the increase of forgetting could be related to the absence of reinforcement. Whatever the reinforcement situation, until the animal was in the goalbox, it could not detect whether there was food in the cup. Even if the pellets had an odor, the alleys could not be distinguished, since one or the other was reinforced according to the subject and no alley was cleaned during a session. Consequently, the extinction operations were probably acting on learned anticipatory running, and not on sensory detection of the absence or presence of pellets in the goalbox. When extinction was used, the decrement in performance appeared earlier, in terms of global performance. When successive trials within the test session are considered, it is quite evident that responding to $\mathrm{S}+$ is slower than that found in Experiment 1. On the first trials, $\mathrm{S}$ - responses showed some differences from $\mathrm{S}+$ responses. Moreover, mean $S-$ running times progressively increased over successive trials, except for the last intervals. This resulted in an increase over trials in the difference between $S+$ and $S-$ running times, although this difference was less than that found with retention. This last effect, which appeared mainly for the two shortest intervals, was somewhat unexpected. It could be considered as a relative relearning related to latent internal or external cues. However, this could be explained by a combination of a general tendency to extinguish due to forgetting related to the time interval and to the generalization of the S- situation. According to the test interval, the respective roles of each of these two factors could be different. A recency effect described by Thomas, Moye, and Kimose (1984) might explain the evolution of $S+$ and $S$ - running times over trials on the two shortest intervals. Changes in reward between training and testing have probably produced interference, but our paradigm does not allow any clear conclusion on this point.

\section{GENERAL DISCUSSION}

Finally, our data constitute a parametric study of the amount of forgetting of an appetitive visual discrimination in BALB/c mice as a function of time. The experimental conditions and the successive discrimination paradigm that we used seemed to show that some factors, such as contextual cues and environmental components, do not play a major role. At the same time, inhibition processes are probably of great importance. Performance was studied using two criteria which gave complementary information. According to both of them, the decay of performance started after the 7th day, and forgetting was nearly complete on the 30 th day. Both retention and extinction were used for testing. A larger decrease of performance was observed with extinction, but the scores after 30 days were the same in both situations. As a function of the test interval, relearning was less sensitive than extinction in that significant effects were observed earlier with the extinction measure. The analysis of performance trial by trial during testing allowed for the separation of initial performance and relearning, at least when testing in Experiment 1. Considering all these elements, it can be hypothesized that the strength of remembering on each test interval should be intermediate between those observed on each of both situations. In other words, the forgetting curves as observed by the discrimination ratio for both situations may represent the envelope of the decay of memory trace in our experimental conditions. As both curves were at the same level on the 30-day interval, this level could independently represent the residual information at this delay for the reinforcement situation.

In conclusion, our data constitute mainly a parametric study that defines a forgetting curve in mice. The data by themselves do not explain the underlying psychological mechanisms. The data seem reliable enough, provide new information concerning this strain of mouse, and constitute a basis upon which to consider studies on the neurobiological bases of long-term retention and forgetting.

\section{REFERENCES}

Brito, G. N. O., Thomas, G. J., \& Stanton, M. E. (1982). Septohippocampal disconnection and Go-NoGo discrimination in rats. Behavioral \& Neural Biology, 34, 427-432.

Coulter, X., Colmer, A. C., \& Campbell, B. A. (1976). Long-term retention of early Pavlovian fear conditioning in infant rats. Journal of Experimental Psychology: Animal Behavior Processes, 2, 48-56.

Deweer, B., Sara, S. J., \& Hars, B. (1980). Contextual cues and memory retrieval in rats: Alleviation of forgetting by a pretest exposure to background stimuli. Animal Learning \& Behavior, 8, 265-272.

Gleitman, H. (1971). Forgetting of long-term memories in animals. In W. H. Honig \& P. H. R. James (Eds.), Animal memory. New York: Academic Press.

HAGGBLOOM, S. J. (1980). Resistance to discrimination and subsequent resistance to extinction as a function of the sequence of partial $\mathrm{S}+$ reward in differential conditioning. Animal Learning \& Behavior, 8 , 441-446. 
Holloway, F. A., \& Wansley, R. A. (1973). Multiple retention deficits at periodic intervals after passive avoidance learning. Science, $\mathbf{8 0}$, 208-210.

Jaffard, R., Destrade, C., Soumireu-Mourat, B., \& Cardo, B (1974). Time-dependent improvement of performance on appetitive tasks in mice. Behavioral Biology, 11, 89-100.

KraEMER, P. J. (1984). Forgetting of visual discriminations by pigeons Journal of Experimental Psychology: Animal Behavior Processes, 10, 530-542.

Messenger, J. B. (1971). Two-stage recovery of a response in sepia. Nature, 232, 202-203.

SARA, S. J. (1973). Recovery from hypoxia and ECS-induced amnesia after a single exposure to training environment. Physiology \& Behavior, 10, 85-89.

SARA, S. J. (1984). Forgetting of a conditioned emotional response and its alleviation by pretest amphetamine. Physiological Psychology, 12, 17-22.
SPEAR, N. E. (1978). The processing of memories: Forgetting and retention. Hillsdale, NJ: Erlbaum.

Steinert, P. A., Infurna, R. N., \& SPEar, N. E. (1980). Long-term retention of conditioned taste aversion in preweanling and adult rats. Animal Learning \& Behavior, 8, 375-381.

Thomas, D. R., \& LoPEz, L. J. (1962). The effect of delayed testing on generalization slope. Journal of Comparative Physiological Psychology, 44, 541-544.

Thomas, D. R., McKelvie, A. R., Ranney, M., \& Moye, T. B. (1981). Interference in pigeons' long-term memory viewed as a retrieval problem. Animal Learning \& Behavior, 9, 581-586.

Thomas, D. R., Moye, T. B., KImose, E. (1984). The recency effect in pigeons' long-term memory. Animal Learning \& Behavior, 12 , 21-28.

(Manuscript received August 14, 1985 revision accepted for publication March 18, 1986.) 